\title{
Spacecraft aims to expose violent hearts of galaxies
}

\section{Low-cost mission will tap into the unexplored upper reaches of the $X$-ray spectrum.}

\section{BY ERIC HAND}

$\mathrm{W}$ ho would have thought that a ringside seat at some of the Universe's most extreme events could come cheap? But by the standards of space-based astronomy, the NuSTAR telescope that NASA plans to launch as early as this month has a modest budget, US\$165 million. Yet it will be sensitive to the high-energy photons produced at the turbulent thresholds of supermassive black holes.

Due to be lofted into orbit by a Pegasus rocket launched in mid-air from a carrier jet, NuSTAR (Nuclear Spectroscopic Telescope Array) is taking aim at an under-explored region of the spectrum. It will detect 'hard' X-rays at 5-80 kiloelectronvolts, an energy range between the softer, lower-energy X-rays probed by telescopes such as the Chandra X-ray Observatory and the $\gamma$-rays measured by satellites such as the Fermi telescope. The spacecraft, which features an array of technical innovations, "will be the first mission that can resolve these high-energy X-rays", says Fiona Harrison, an astronomer at the California Institute of Technology in Pasadena and the mission's principal investigator.

Hard X-rays are notoriously difficult to focus, because they tend to penetrate rather than reflect off mirrors, even those coated with dense metals such as gold and iridium. So Harrison and her colleagues have developed a novel material: a sandwich of hundreds of thin

\section{GOING TO EXTREMES}

Compared with other X-ray telescopes, NuSTAR has a larger collecting surface at higher energies.

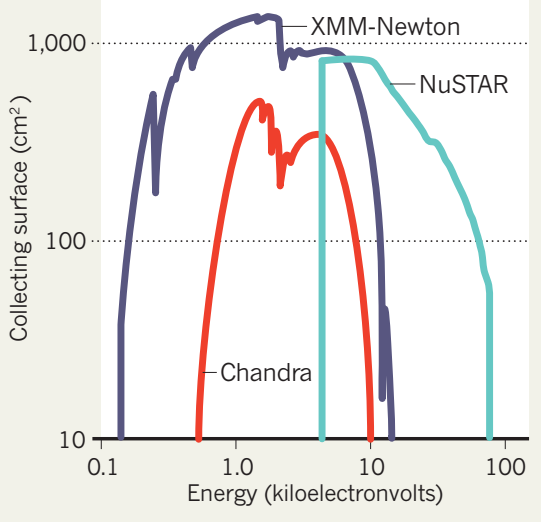

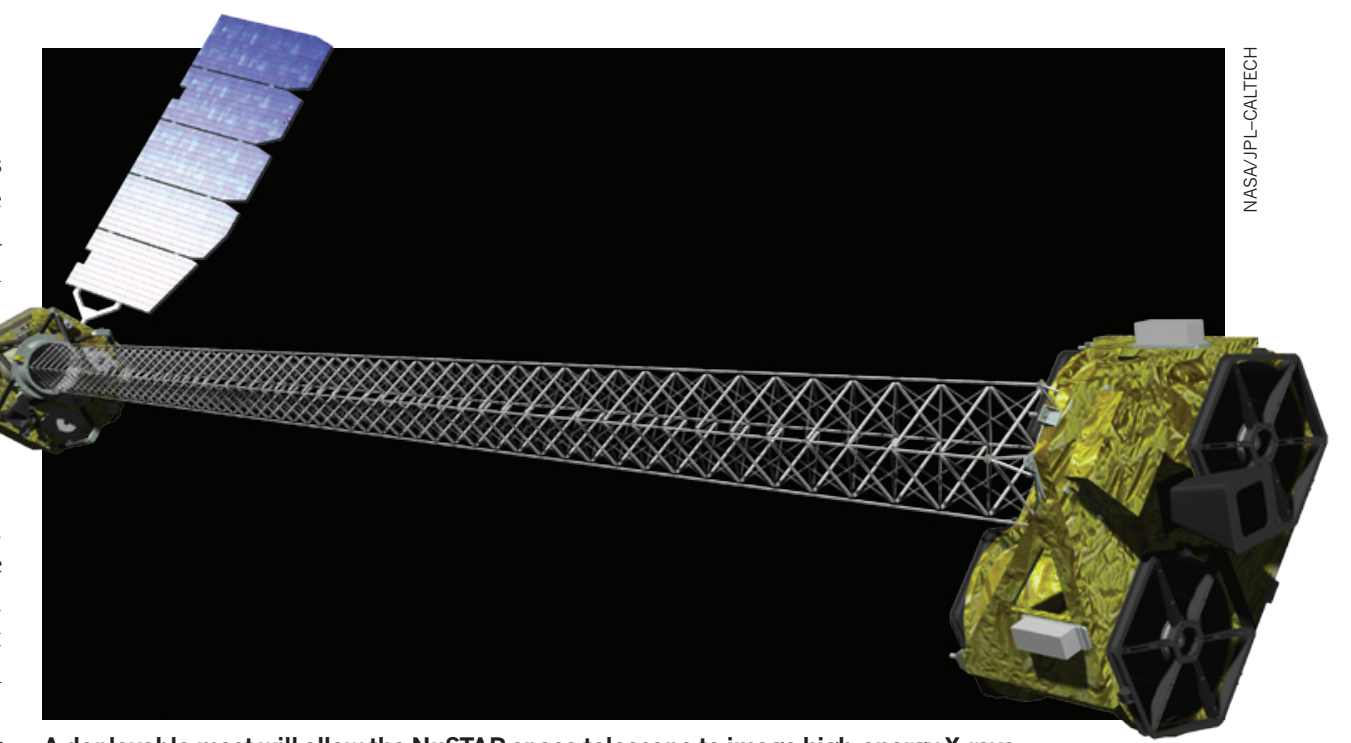

A deployable mast will allow the NuSTAR space telescope to image high-energy X-rays.

metallic films. The thickness of each layer is tuned to reflect photons of a specific energy. Each layer reflects a small number of photons, but they add up to produce a strong reflection. "That's the key technical breakthrough," says Harvey Tananbaum, an astronomer at the Harvard-Smithsonian Center for Astrophysics in Cambridge, Massachusetts, and director of the Chandra telescope. "Nobody has been there with this kind of sensitivity."

Still, the X-rays are so powerful that they can be reflected only at glancing angles, and the mirrors must therefore be arranged in conical shells, like nested Russian dolls, which funnel the high-energy photons into a detector at the end of the structure. With 133 of these mirror shells in each of two units, NuSTAR will be more sensitive than the Chandra telescope (see 'Going to extremes'), which has only four mirror shells - although, because they are stiffer and more finely polished, Chandra can perceive objects in greater detail.

Grazing-incidence optics means that a long focal length is needed - and, conventionally, a bulky, costly spacecraft. The $\$ 2$-billion Chandra could barely squeeze into the cargo bay of the Space Shuttle. NuSTAR, at 10 metres, will be just as long - but it has to fit on the much smaller, cheaper Pegasus launcher. The solution: the telescope is built around a folded-up truss that will grow to full length once NuSTAR is deployed in low-Earth orbit. For astronomers, the 26 minutes while NuSTAR unpacks itself will be the most anxious phase of the mission.

The telescope will focus on active galactic nuclei (AGN), the luminous hearts of distant galaxies, in which highly energized particles fling off X-rays as they whirl around supermassive black holes. Because NuSTAR is sensitive to the most penetrating X-rays, it should be able to spot several hundred new AGN that are shrouded from other detectors by dust and hot gas. That should help astronomers to get a handle on how big and common AGN are, says Harrison, which in turn will help to illuminate a major cosmic puzzle: what role do they have in the growth and evolution of their host galaxies?

The European Space Agency's XMMNewton mission, another large X-ray telescope, will join forces with NuSTAR to probe AGN. The two spacecraft will look for distortions in the X-ray spectra resulting from the spin of the central black hole. These spin rates, in turn, could offer insight into how the black holes in some AGN grew to masses of billions of Suns whether by swallowing other AGN in galactic mergers or by steadily accumulating material from the host galaxy. XMM-Newton is devoting $10 \%$ of its guaranteed observation programme time to joint observations with NuSTAR.

"It shows how important this mission is to the community," says Norbert Schartel, the project scientist for XMM-Newton at the European Space Astronomy Centre in Madrid. 